\title{
Total energy conservation in ALE schemes for compressible flows
}

\author{
Alain Dervieux ${ }^{1}$ - Charbel Farhat ${ }^{2}$ - Bruno Koobus ${ }^{3}$ \\ Mariano Vázquez 4
}

1. INRIA, Projet Tropics, BP 93, 06902 Sophia-Antipolis cedex, France

2. Dept of Mechanical Engineering and Institute for Computational and Mathematical Engineering, Stanford University, Building 500

Stanford CA 94305-3035, USA

3. Mathématiques, université de Montpellier II, CC.051

34095 Montpellier, France

4. CASE Dpt. Barcelona Supercomputing Center BSC-CNS C/ Gran Capitán 2

2 a Planta 08034 Barcelona, Spain

ABSTRACT. The numerical prediction of interaction phenomena between a compressible flow model with a moving domain and other physical models requires that the work performed on the fluid is properly translated into total fluid energy variation. We present a numerical model relying on an Arbitrary Lagrangian-Eulerian (ALE) unstructured vertex-centered finite volume that satisfies this condition together with the Geometric Conservation Law. We apply this numerical scheme to the solution of a $3 D$ fluid-structure interaction problem. The results are contrasted with those obtained by the energy non-conservative counterpart.

RÉSUMÉ. La prédiction numérique de l'interaction entre un fluide dans un domaine mobile et un autre modèle nécessite que le travail appliqué à sa frontière soit traduit en une variation appropriée de son énergie interne. On présente un modèle numérique reposant sur une formulation Arbitraire Lagrange Euler (ALE) en volumes finis non structurés et centrés sommet satisfaisant cette propriété ainsi que la Loi de Conservation Géométrique. Le schéma est appliqué à un problème d'interaction fluide-structure $3 D$. Les résultats sont mis en contraste avec ceux obtenus par un schéma analogue non conservatif pour l'énergie totale.

KEYWORDS: Arbitrary Lagrangian Eulerian, compressible flow, spatial discretization, discrete geometric conservation law, total energy conservation.

MOTS-CLÉS : Euler Lagrange Arbitraire, écoulement compressible, discrétisation spatiale, loi de conservation géométrique discrète, conservation d'énergie totale.

DOI:10.3166/EJCM.19.337-363 @ 2010 Lavoisier, Paris 


\section{Introduction}

The motivation of this study is the improvement of fluid numerical models in moving domain with the purpose of application to complex interaction problems such as fluid-structure unsteady interaction. Let us explain in detail the problems that arise in this kind of compound dynamical systems by concentrating on a decisive issue: the numerical prediction of the unsteady interaction between a compressible flow and a structure requires that the global numerical scheme satisfies some conservation properties.

To start with, fixed mesh calculations compulsorily require conservation formulations (mass, momentum, energy) for the fluid model. Two main motivations for this choice follows. Firstly, physical soundness of many computations requires that important extensive quantities are conserved during time evolution. Secondly, a certain class of conservative schemes allows the application of the Lax-Wendroff convergence theorem towards weak solution. The effect of this convergence appears not only in the capture of shocks but also in very irregular meshes. In the case of moving meshes, the Arbitrary Lagrangian Eulerian $(A L E)$ formulation of Hirt, Amsden and Cook (Hirt et $a l ., 1974)$ gave an answer to the first above conservation issue.

A second notion of conservation arose rather early for moving mesh methods. The Geometric Conservation Law (GCL), introduced in (Thomas et al., 1979), and its discrete versions $(D G C L)$ ensure that a uniform flow is not perturbed by approximation errors during the mesh motion. Many important arguments can be invoked for the design of schemes respecting this condition.

- It was first shown, for example in (Lesoinne et al., 1996)(Koobus et al., 1999), that its practical impact on accuracy and stability is of paramount importance.

- Since degree zero polynomial solutions are exactly obtained, the GCL can be understood as a consistency condition (of Bramble-Hilbert type). A mathematical analysis has demonstrated the role of the GCL for time-space accuracy order for ALE schemes (Guillard et al., 2000).

- Also the GCL guarantees that the uniform solution will not show spurious undershoot or overshoot, that is, erroneous extrema. In (Farhat et al., 2001; Cournède et al., 2006), it is proved that the discrete GCL is a sufficient condition in order that some schemes satisfy the maximum principle for passive species. The DGCL is then a nonlinear stability condition.

A third important conservation aspect in the discretization of fluid-structure interaction is the energy conservation. A theoretical review for different models is, for instance, that of (Grandmont et al., 2000). From a numerical point of view, the global energy conservation issue can be crucial, particularly in problems where the energy transfered to the structure is produced by thermodynamic effects. Two main sources of spurious loss of energy can be identified and distinguished:

a. The time advancing method. Basically, the time integration can be done in three different ways. Explicitly (1), implicitly and loosely coupled (2) or implicitly 
and strongly coupled (3), which is also called monolithic approach (see for example (Leyland et al., 2000)). Case (1) has often proven to be too expensive in terms of CPU time. On the implicit side, up to our knowledge, only a small subset of monolithic schemes (3) allows a strictly energy conservation integration. The drawback is again their very high computational cost. For that reason, loosely coupled implicit schemes (2) are the most widespread choice. Among them, recently proposed staggered algorithms satisfy the energy conservation through the interface not exactly but up to second order in time (or third order: see the paper (Piperno et al., 2000)). Energy conservative schemes are also addressed in (Mani, 2003) and (Le Tallec et al., 2005).

$b$. The space integration. The spatial-oriented study of the energy budget for moving boundary problems, with coupling or not, and solved by means of ALE schemes can be carried out following two lines.

The first line concerns the work relationship between coupled fluid and structure: is the work performed on the fluid by the moving interface the same done by the solid through this interface? Since, in general, the structural model does not involve an energy equation, this kind of study concerns kinetic energy in the coupling of moment and displacement equations.

This line is considered in papers like (Farhat et al., 1998) or (Piperno et al., 2000). Both systems (fluid, structure) are treated by their own equations, and a third one is included which governs the spatial fluid grid movement. All three are coupled through the moving interface. In (Farhat et al., 1998), the space discretization issue is analyzed, focusing on the load forces distribution at the interface, as seen either by the fluid or the solid. In (Piperno et al., 2000), several ideas for the implicit time integration scheme are proposed, based on a staggered formulation.

The second line concerns the work-energy relationship within the fluid, regardless the structure: is the work performed on the fluid by the moving interface properly translated in a variation of the total energy of the fluid? And this concerns essentially compressible fluids.

The total energy conservation issue is important in many engineering applications related to energy, for example in thermal engines or power plant turbines. It is considered in a series of papers (Fanion et al., 2000; Le Tallec et al., 2000; Fernandez et al., 2002), in which it is used as a stability criterion for building a transpiration condition.

The present paper also focuses on that second line with a special examination of boundary conditions for ALE. The action of the moving boundary on the fluid system produces a variation in its energy status. It is clear for the continuum problem, but the discrete form remains full of questions. The core of this problem remains in the flow equations discretization alone. How can an ALE scheme cope with this equivalence? How can the work performed on the system be properly defined? How does the fluid energy, defined as an extensive system property and related to the work, change? Are there fully energy preserving schemes that also satisfy the other conservation relations 
(mass, momentum) and the Geometric Conservation Law? Once the questions are solved, their conclusions can be easily adapted to the coupled schemes.

The paper is organized as follows. First, the continuum problem is set: the Euler equations for compressible inviscid flow are written down and the energy budget is presented. This is done in a fixed Eulerian reference system but a moving domain $\Omega(t)$ is considered. Then, the flow equations are discretized in space with unstructured finite volume, following an ALE approach which deals with the moving boundary difficulty, and satisfies also the GCL. That section follows (Nkonga et al., 1994), keeping and extending the notations of this reference. The variables approximation and integral quardrature on the surface is geometrically complex and will be carefully described. Next, the analysis on the energy conservation issue is conducted, resulting in a new ALE scheme. Then, we apply this scheme to a piston model flow and to a flutter analysis of the AGARD Wing 445.6 in order to contrast the results with those obtained by a counterpart of this scheme which does not satisfy the total fluid energy conservation. Finally, we conclude this paper in Section 6.

\section{Notations and conventions}

The following notations and conventions are here adopted:

NC1: Variables notation:

- Scalars: $\rho, p, E$, etc.

- Vectors: $\boldsymbol{u}, \boldsymbol{U}, \boldsymbol{x}$, etc.

- Tensors: $\tau, \sigma$, etc. Space indicial notation for vectors and tensors is here scarcely preferred, only for neatness criteria.

- Extensive variables: $\mathcal{E}, \mathcal{V}$, etc., in upper case script style.

- Variables defined at nodes: $\rho_{i}, \boldsymbol{u}_{i}$, etc. Subindices always refer to nodal number, unless otherwise stated.

- Variables defined at cell boundaries: $\kappa_{i j}, \boldsymbol{\nu}_{i j}$, etc., labelled with two subindices.

- Variables defined at the domain boundaries: $\widehat{\boldsymbol{u}}, \widehat{p}, \widehat{\boldsymbol{\beta}_{i j}}$, etc., labelled or not.

Einstein's summation on repeated indices (index contraction) is never assumed unless otherwise stated.

NC2: Considering integrals, the following notation is adopted, unless otherwise stated. For any field $f$,

$$
\int_{A} f:=\int_{A} f d A
$$

where $A$ can be volume, surface or time interval, and $d A$ labels the corresponding differential. 


\section{The physical problem: the Euler equations for compressible flow}

The physical problem under study is the inviscid compressible flow. Its physics is modelled by the Euler equations, basically the Navier - Stokes set without viscous terms. For compressible flows, this set of equations comprises transport equations for the mass, the momentum and the energy (see for instance (Landau et al., 1987)). Due to compressibility pressure $p$, density $\rho$ and temperature $T$ changes are related through a state equation, which couples the energy budget with the mass and force balances.

Suppose a fluid is contained in a given spatial domain $\Omega \subset \mathbb{R}^{n}$, where $n$ is the space dimension. All the variables that describe its behaviour are then functions of position $\boldsymbol{x}$ and time $t$, being $t \in[0, \infty)$. In conservative form, the unknowns of the Euler equations are respectively the density $\rho$, the momentum $\boldsymbol{U}=\rho \boldsymbol{u}$, and the total energy per unit volume $E=\rho e$. The velocity vector is noted as $\boldsymbol{u}$ and $e=e^{o}+\frac{1}{2} u^{2}$ is the total energy per unit mass, being $e^{o}$ the fluid's internal energy and $u^{2}=\boldsymbol{u} \cdot \boldsymbol{u}$. Hence, the conservation form is

$$
\begin{aligned}
& \frac{\partial \rho}{\partial t}+\frac{\partial}{\partial x_{i}}\left(U_{i}\right)=0 \\
& \frac{\partial U_{j}}{\partial t}+\frac{\partial}{\partial x_{i}}\left(u_{i} U_{j}+p \delta_{i j}\right)=0 \\
& \frac{\partial E}{\partial t}+\frac{\partial}{\partial x_{i}}\left(u_{i}(E+p)\right)=0
\end{aligned}
$$

where the subindices note space components, i.e. $i, j=1, \ldots, n$ and repeated indices contract. As viscous forces are absent, the stress tensor is $\sigma_{i j}=-p \delta_{i j}$. To close the equations, a state law is needed: the ideal gas state law $p=\rho R T$ for instance. Under this hypothesis, the internal energy is $e^{o}=C_{\mathrm{v}} T . R$ is the universal gas constant and $C_{\mathrm{v}}$ and $C_{\mathrm{p}}$ are the specific heat at constant volume and at constant pressure respectively.

In addition to [1], a set of properly defined boundary (discussed later) and initial conditions are needed to tackle the solution of the problem. Initial conditions are of the kind

$$
\begin{array}{lr}
\boldsymbol{u}(\boldsymbol{x}, 0):=\boldsymbol{u}^{0}(\boldsymbol{x}), & \text { for all } \boldsymbol{x} \in \Omega \\
\rho(\boldsymbol{x}, 0):=\rho^{0}(\boldsymbol{x}), & \text { for all } \boldsymbol{x} \in \Omega \\
T(\boldsymbol{x}, 0):=T^{0}(\boldsymbol{x}), & \text { for all } \boldsymbol{x} \in \Omega
\end{array}
$$

and the rest of the initial variables can be derived from them. 


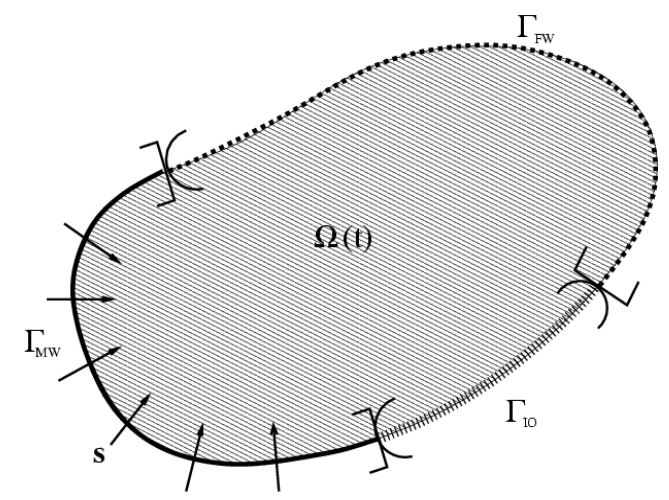

Figure 1. Sketch of the generic problem

\subsection{Moving domains}

The core of the fluid-structure interaction problems is the boundary condition that couples both systems. By its motion, the solid performs work against the internal forces of the fluid, producing a change in its energetic status. To focus on this issue, a generic problem is considered, sketched in Figure 1. Suppose that $\Omega=\Omega(t)$, whose boundary is divided in three disjoint subsets: a fixed wall $\Gamma_{\mathrm{FW}}$ and a moving wall $\Gamma_{\mathrm{MW}}(t)$, and an input / output $\Gamma_{\mathrm{IO}}$ boundary (additionally, let all boundaries be adiabatic ones):

$$
\partial \Omega(t)=\Gamma_{\mathrm{FW}} \cup \Gamma_{\mathrm{IO}} \cup \Gamma_{\mathrm{MW}}(t) .
$$

Under these assumptions, the boundary conditions should be compatible with both the wall (moving or not) impermeability and adiabaticity, strongly imposed as Dirichlet's (on some velocity components) or weakly imposed as Neumann's (on the traction or the heat flow). Hereafter, $s$ denotes the velocity distribution for all the points belonging to the moving wall measured in a fixed Eulerian frame. For the sake of clarity, in the rest of the analysis, fixed boundaries like $\Gamma_{\mathrm{FW}}$ and $\Gamma_{\mathrm{IO}}$ are represented by $\Gamma$ and moving walls like $\Gamma_{\mathrm{MW}}(t)$ by $\Gamma(t)$.

The conservation form of the flow equations is derived from a simple fact: some important properties are conserved as fluid gets in and out of a given volume. To evaluate this balance, all of the differential equations [1] can be integrated in space as follows. Let us write them generically as:

$$
\frac{\partial v}{\partial t}+\nabla \cdot \boldsymbol{F}(v)=0
$$


where $v$ and $\boldsymbol{F}$ represents the transported quantity and its flux respectively. Integrated in space, (recall $N C 2$ notation) we get:

$$
\int_{\Omega} \frac{\partial v}{\partial t}+\int_{\partial \Omega} \boldsymbol{F} \cdot \boldsymbol{n}=0
$$

after using the divergence Gauss' theorem. The vector $\boldsymbol{n}$ is the unit exterior normal to the boundary $\partial \Omega$. In this way, extensive flow quantities can be studied. When a locally defined variable like $v$ is integrated over the volume $\Omega$ under study, we obtain an extensive quantity $\mathcal{V}$ :

$$
\mathcal{V}=\int_{\Omega} v
$$

The evolution of the extensive quantities is modeled by equations like [4]. If in [4], the integration domain is changing with time, i.e. $\Omega=\Omega(t)$, the partial time derivative can be taken out of the integral by means of the so called Reynolds' formula:

$$
\frac{d \mathcal{V}}{d t}=\frac{d}{d t}\left(\int_{\Omega(t)} v\right)=\int_{\Omega(t)} \frac{\partial v}{\partial t}+\int_{\partial \Omega(t)} v \boldsymbol{s} \cdot \boldsymbol{n} .
$$

By using [5] in [4], we obtain

$$
\frac{d \mathcal{V}}{d t}+\int_{\partial \Omega(t)} \boldsymbol{F} \cdot \boldsymbol{n}-\int_{\partial \Omega(t)} v \boldsymbol{s} \cdot \boldsymbol{n}=0
$$

which is the basis of any ALE formulation, a well-suited idea for solving flow problems with moving boundaries. Equation [6] is the key to understand the conservation principles under study in this paper: any ALE scheme should satisfy the energy conservation and the so-called geometric conservation law. Both are introduced in the next two sections.

\subsection{The continuous energy budget}

We recall now in what sense total energy is conserved for an Euler flow set in a moving domain. We start from [5], which we restrict to the total energy equation, as written in the set [1], and which we integrate over the whole domain. This will give the amount of energy introduced to (or extracted from) the fluid system by the solid via the mechanical action of the moving boundary. So let $v$ in [5] be replaced by $v=E=\rho e^{o}+\rho \frac{u^{2}}{2}$, the total energy. Then, its extensive counterpart is

$$
\mathcal{E}=\int_{\Omega} E
$$


which in turn satisfies the Reynolds' formula [5]

$$
\int_{\Omega(t)} \frac{\partial}{\partial t}\left(\rho e^{o}+\rho \frac{u^{2}}{2}\right)=\frac{d \mathcal{E}}{d t}-\int_{\partial \Omega(t)}\left(\rho e^{o}+\rho \frac{u^{2}}{2}\right) \boldsymbol{s} \cdot \boldsymbol{n} .
$$

Therefore, the energy transport equation can be written as

$$
\begin{aligned}
\frac{d \mathcal{E}}{d t}= & -\int_{\partial \Omega(t)}\left(\rho e^{o}+\rho \frac{u^{2}}{2}\right) \boldsymbol{u} \cdot \boldsymbol{n}-\int_{\partial \Omega(t)} p \boldsymbol{u} \cdot \boldsymbol{n} \\
& +\int_{\partial \Omega(t)}\left(\rho e^{o}+\rho \frac{u^{2}}{2}\right) \boldsymbol{s} \cdot \boldsymbol{n} .
\end{aligned}
$$

Since the impermeability condition

$$
\begin{aligned}
& \left.\boldsymbol{u}\right|_{\Gamma_{\mathrm{MW}}} \cdot \boldsymbol{n}=\boldsymbol{s} \cdot \boldsymbol{n} \\
& \left.\boldsymbol{u}\right|_{\Gamma_{\mathrm{FW}}} \cdot \boldsymbol{n}=0
\end{aligned}
$$

is assumed both on the moving and fixed walls, the normal flow velocity $\boldsymbol{u} \cdot \boldsymbol{n}$ is there equal to the normal solid wall velocity $\boldsymbol{s} \cdot \boldsymbol{n}$. This additional condition is used to cancel some terms in [8] on the moving boundary, which now becomes

$$
\frac{d \mathcal{E}}{d t}=-\int_{\Gamma_{\mathrm{IO}}}\left(\rho e^{o}+\rho \frac{u^{2}}{2}\right) \boldsymbol{u} \cdot \boldsymbol{n}-\int_{\Gamma_{\mathrm{IO}}} p \boldsymbol{u} \cdot \boldsymbol{n}-\int_{\Gamma_{\mathrm{MW}}} p \boldsymbol{s} \cdot \boldsymbol{n}[10]
$$

The energy variation in time consists of three boundary terms, shown in Equation [10]. The first one is the total energy per time unit convected in or out of the domain through the inflow and outflow. The second one is the work per time unit performed by the fluid against this in/outcoming energy. The third one represents the mechanical action of the solid on the fluid. It is the work per time unit performed on the fluid by the moving wall. Note that all three integrand are co-linear with the normal velocity and are present regardless either viscosity or compressibility.

Equation [10] has been calculated from the space integration of the total energy transport equation for the fluid. In turn, it can be integrated in time to get the energy variation $\Delta \mathcal{E}$

$$
\left.\Delta \mathcal{E}\right|_{t_{1}} ^{t_{2}}=\int_{t_{1}}^{t_{2}} \frac{d \mathcal{E}}{d t}
$$

between times $t_{1}$ and $t_{2}$ (being $t_{1}<t_{2}$ ). It should satisfy

$$
\left.\Delta \mathcal{E}\right|_{t_{1}} ^{t_{2}}=\left.\Delta \mathcal{Q}\right|_{t_{1}} ^{t_{2}}+\left.W\right|_{t_{1}} ^{t_{2}}=\left.W\right|_{t_{1}} ^{t_{2}}
$$


where $\left.\Delta \mathcal{Q}\right|_{t_{1}} ^{t_{2}}$ holds for the thermical contribution to the gas from outside, and $\left.W\right|_{t_{1}} ^{t_{2}}$ for work performed by the external forces on the fluid system between $t_{1}$ and $t_{2}$. We assume that $\Delta \mathcal{Q}=0$. As highlighted in the introduction, Equality [12] is the key of the present work: trivial in the continuum, it should be analyzed when time and space discretizations are envisaged to solve the problem. $\left.W\right|_{t_{1}} ^{t_{2}}$ can be split as follows:

$$
\left.W\right|_{t_{1}} ^{t_{2}}=\left.\left(W_{\mathrm{IO}}+W_{\mathrm{MW}}\right)\right|_{t_{1}} ^{t_{2}}
$$

This includes the three contributions of Equation [10]. Those coming from the input/output domain boundary, grouped in $W_{\text {IO }}$, also appear when all boundaries are fixed and therefore $W_{\text {IO }}$ presents no particular difficulties in an ALE context. The differential fact is now the work performed by the moving wall on the fluid, noted as $W_{\mathrm{MW}}$.

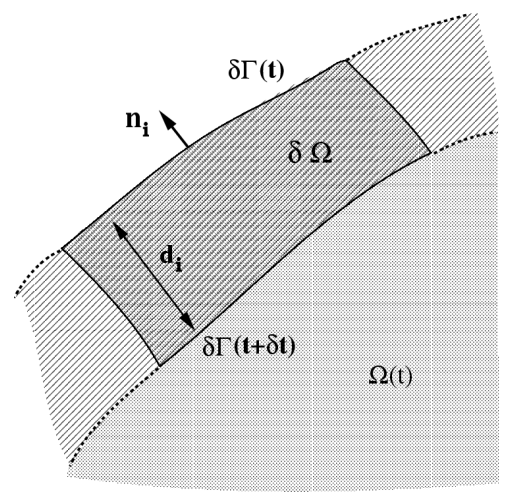

Figure 2. Work performed by the moving wall

While the right end side of [11] is calculated as a domain integral of the energy transport equation, the right hand side of [12] can be evaluated from outside (with respect to the fluid), considering the motion of $\Gamma(t)$, the work spent in this motion and the energy-work interchanges in inputs and outputs. From now on, we will focus only on what happens with the moving part of the boundary, leaving aside $W_{\text {IO }}$. Let suppose a small time increment $\delta t$ for which the moving wall goes from $\Gamma(t)$ to $\Gamma(t+$ $\delta t)$. Let $f_{i}$ be the force per unit surface, $\delta w$ its performed work and $d_{i}$ the distance between $\delta \Gamma(t)$ and $\delta \Gamma(t+\delta t)$ which are very small portions of $\Gamma(t)$ and $\Gamma(t+\delta t)$ respectively (Figure 2). On these grounds, it can be defined

$$
\begin{aligned}
& \boldsymbol{f}=\boldsymbol{\sigma} \cdot \boldsymbol{n} \\
& \boldsymbol{d}=\boldsymbol{s} \delta t .
\end{aligned}
$$

where $\sigma$ is the stress tensor defined above. Then,

$$
\delta w=\boldsymbol{d} \cdot \boldsymbol{f}=\boldsymbol{d} \cdot(\boldsymbol{\sigma} \cdot \boldsymbol{n})=(\delta t \boldsymbol{s}) \cdot(\boldsymbol{\sigma} \cdot \boldsymbol{n})
$$


which yields

$$
\delta W=\delta \Gamma \delta t \boldsymbol{s} \cdot \boldsymbol{\sigma} \cdot \boldsymbol{n} .
$$

Integrated in time (between $t_{1}$ and $t_{2}$ ) and surface (over the whole $\partial \Omega(t)$, which corresponds to $\Gamma(t))$, it gives

$$
\left.W\right|_{t_{1}} ^{t_{2}}=\int_{t_{1}}^{t_{2}} \int_{\partial \Omega(t)} \boldsymbol{s} \cdot \boldsymbol{\sigma} \cdot \boldsymbol{n} d \Gamma d t .
$$

In few words: regardless the in/outlets contribution, the left hand side of [12] (LHS[12], from now on) is Equation [10] integrated in the given time interval, that is to say [11], and the right hand side (RHS[12]) is Equation [16]. The LHS[12] is calculated in an Eulerian formulation for a moving domain, starting from total energy general conservation principles for fluid dynamics. It describes what happens "on the fluid side". The RHS[12] is calculated as the work introduced in the system via the moving boundaries, it is "on the solid side" (recall we have left aside $W_{\text {IO }}$ ).

\section{Discrete standpoint}

The DGCL for a finite volume formulation on tetrahedral, non-structured grids, extended to an ALE formulation has been proposed in (Nkonga et al., 1994). We recall in Sec. 3.1, 3.2, 3.3 the main ingredients of this work in order to show that this DGCL formulation is sufficient for total energy conservation, except at boundary level. Sec. 3.5 examines the energy conservation issue for boundary fluxes.

\subsection{Spatial discretization of the Euler equations}

Equations [1] can be written in a compact form. For each component,

$$
\frac{\partial v}{\partial t}+\nabla \cdot \boldsymbol{F}(v)=0
$$

taking

$$
\begin{aligned}
v & =\left(\rho, \rho u_{1}, \rho u_{2}, \rho u_{3}, \rho e\right)^{T} \\
F_{k}(v) & =\left(U_{k}, u_{k} U_{1}+p \delta_{k 1}, u_{k} U_{2}+p \delta_{k 2}, u_{k} U_{3}+p \delta_{k 3}, u_{k}(\rho e+p)\right)^{T}
\end{aligned}
$$

for a tridimensional problem, the subindex $k$ running through the three space coordinates.

The weak form of [17] is discretized using a nodal centered finite volume method. The computational domain is discretised into $\Omega_{\mathrm{h}}^{0}$, a conformal finite-element mesh 


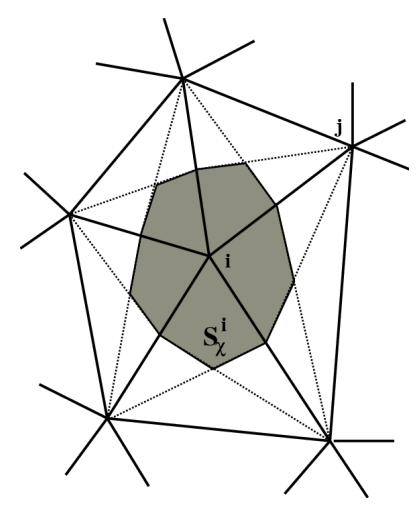

Figure 3. FV support or control cell $C^{i}$ corresponding to node $i(2 D$ case)

made of triangles (2D) or tetrahedra (3D). A dual finite-volume partition of $\Omega_{\mathrm{h}}^{0}$ is obtained by building around each vertex $i$ a dual cell $C^{i}$ limited by medians (2D, see Figure 3 ) and median plans in 3D. The finite-volume basis function space is then made of characteristic functions of these cells:

$$
\phi=\chi^{i}(\boldsymbol{x})= \begin{cases}1 & \text { if } \boldsymbol{x} \in C^{i} \\ 0 & \text { otherwise }\end{cases}
$$

In this way, the control cells cover completely $\Omega_{\mathrm{h}}^{0}$ :

$$
\Omega_{\mathrm{h}}^{0}=\bigcup_{i=1}^{n_{c}} C^{i}
$$

Then, the weak form of [17] is integrated in the partitioned $\Omega_{\mathrm{h}}^{0}$ and using $\chi^{i}$ following these ideas. For each node, we have

$$
\int_{C^{i}} \frac{\partial v}{\partial t}+\int_{\partial C^{i}} \boldsymbol{n}_{i} \boldsymbol{F}(v)=0 .
$$

Many options are then possible for the integration at cell boundary. We refer for example to (Cournède et al., 2006).

\subsection{Discretization of moving domains: ALE method}

Let us consider a smooth bijective mapping $\pi(t)$ depending on time and equal to identity at time $t=0$. Defining this mapping is equivalent to defining a velocity field $\boldsymbol{\beta}$ and moving each point of the space with this velocity. Let for any time $\Omega_{\mathrm{h}}(t)$ the 
triangulation (tetrahedrisation in 3D) obtained by applying the mapping $\pi(t)$ to any vertex of $\Omega_{\mathrm{h}}^{0}$. It is enough to known the trajectories of each vertex, starting from a vertex of $\Omega_{\mathrm{h}}^{0}$. This is also equivalent to know for any time $t$ the velocity $\boldsymbol{\beta}(i, t)$ at vertex $i$ of $\Omega_{\mathrm{h}}^{t}$. In order to ensure than any segment or plan of the initial mesh will stay at ant time resp. a serment or a plan, we consider the linear interpolation of vertex values $\boldsymbol{\beta}(i, t)$ to any element of mesh $\Omega_{\mathrm{h}}^{t}$. For this discrete deformation velocity, we keep the notation $\boldsymbol{\beta}(t)$. In $\Omega_{\mathrm{h}}^{t}$ we build the dual cells:

$$
\Omega_{\mathrm{h}}(t)=\bigcup_{i=1}^{n_{c}} C^{i}(t)
$$

The flux balance writes now:

$$
\frac{d \mathcal{V}^{i}}{d t}+\int_{\partial C^{i}(t)} \boldsymbol{F}(v) \cdot \boldsymbol{n}_{i}-\int_{\partial C^{i}(t)} v \boldsymbol{\beta} \cdot \boldsymbol{n}_{i}=0
$$

where

$$
\frac{d \mathcal{V}^{i}}{d t}=\frac{d}{d t}\left(\int_{C^{i}(t)} v\right)
$$

For each interior cell $C^{i}(t)$, with its corresponding set $V(i)$ of neighboring cells,

$$
\partial C^{i}(t)=\bigcup_{j \in V(i)} \partial C^{i j}(t)
$$

where $\partial C^{i j}(t)$ represents the interface shared by cells $C^{i}(t)$ and $C^{j}(t)$. Then, [21] can be written as:

$$
\frac{d \mathcal{V}^{i}}{d t}+\sum_{j \in V(i)} \int_{\partial C^{i j}(t)} \boldsymbol{F}(v) \cdot \boldsymbol{n}_{i j}-\sum_{j \in V(i)} \int_{\partial C^{i j}(t)} v \boldsymbol{\beta} \cdot \boldsymbol{n}_{i j}=0 .
$$

In order to evaluate this integral, following (Farhat et al., 2001), let us define

$$
\boldsymbol{\nu}_{i j}(t)=\frac{1}{\left|\partial C_{i j}(t)\right|} \int_{\partial C_{i j}(t)} \boldsymbol{n}_{i j}(t) d s
$$

and

$$
\kappa_{i j}(t)=\frac{1}{\left|\partial C_{i j}(t)\right|} \int_{\partial C_{i j}(t)} \boldsymbol{\beta}(t) \cdot \boldsymbol{n}_{i j}(t) d s .
$$

$\boldsymbol{\nu}_{i j}(t)$ is the mean normal corresponding to $\partial C_{i j}(t)$ and $\kappa_{i j}(t)$, the mean normal mesh velocity projection for the same cell interface (the full meaning of this "mean" will be 
grasped below, when it becomes also a temporal one). Then we get an integral ALE semi-discretization of the conservation law:

$$
\frac{d \mathcal{V}^{i}}{d t}+\sum_{j \in V(i)}\left|\partial C_{i j}(t)\right| \Phi\left(v_{i}, v_{j}, \boldsymbol{\nu}_{i j}(t), \kappa_{i j}(t)\right)=0
$$

where $\Phi$ is a numerical flux function, typically an approximate Riemann solver, with mesh velocity normal component $\kappa_{i j}(t)$ and with mean value of unknown $v$ over cell $i$ denoted by $v_{i}$. In particular it satisfies the following consistency condition:

$$
\Phi(v, v, \nu, \kappa)=\boldsymbol{F}(v) \cdot \boldsymbol{\nu}-\kappa v .
$$

\subsection{Discrete Geometric Conservation Law}

Consider now a time discretization of the above formula. Up to first order, $v_{i}$ can be taken as constant within each cell. Then, if the volume of the partition's cell $C_{i}(t)$ is $\left|C_{i}(t)\right|$,

$$
\mathcal{V}_{i}(t)=\left|C_{i}(t)\right| v_{i}(t)
$$

The $\theta$-parameterized Euler time advancing yields

$$
\begin{aligned}
\left|C_{i}^{n+1}\right| v_{i}^{n+1}= & \left|C_{i}^{n}\right| v_{i}^{n} \\
& -\Delta t \theta \sum_{j \in V(i)}\left|\partial \bar{C}_{i j}\right| \Phi\left(v_{i}^{n+1}, v_{j}^{n+1}, \overline{\boldsymbol{\nu}}_{i j}, \bar{\kappa}_{i j}\right) \\
& -\Delta t(1-\theta) \sum_{j \in V(i)}\left|\partial \bar{C}_{i j}\right| \Phi\left(v_{i}^{n}, v_{j}^{n}, \overline{\boldsymbol{\nu}}_{i j}, \bar{\kappa}_{i j}\right)
\end{aligned}
$$

where the overlines mean that time averaged values are taken. According to the Geometric Conservation Law principle, a uniform solution is exactly preserved when time-advanced by the numerical scheme. Assume the above system able to reproduce a constant solution $v^{n}=v^{n+1}=v^{*}$, it should satisfy:

$\left|C_{i}^{n+1}\right| v_{i}^{*}=\left|C_{i}^{n}\right| v_{i}^{*}-$

$\Delta t \theta \sum_{j \in V(i)}\left|\partial \bar{C}_{i j}\right| \Phi\left(v_{i}^{*}, v_{j}^{*}, \overline{\boldsymbol{\nu}}_{i j}, \bar{\kappa}_{i j}\right)-\Delta t(1-\theta) \sum_{j \in V(i)}\left|\partial \bar{C}_{i j}\right| \Phi\left(v_{i}^{*}, v_{j}^{*}, \overline{\boldsymbol{\nu}}_{i j}, \bar{\kappa}_{i j}\right)$.

Invoking the consistency condition for $\Phi$ and the fact that the cells remain closed during the motion, which writes:

$$
\sum_{j \in V(i)}\left|\partial \bar{C}_{i j}\right| \overline{\boldsymbol{\nu}}_{i j}=0
$$


we see that this gives the usual Discrete Geometric Conservation Law (DGCL):

$$
\left|C_{i}^{n+1}\right|=\left|C_{i}^{n}\right|-\Delta t \sum_{j \in V(i)}\left|\partial \bar{C}_{i j}\right| \bar{\kappa}_{i j} .
$$

As stated in papers like (Nkonga et al., 1994), (Farhat, 1995b) or (Dubuc et al., 2000), the DGCL becomes a design condition to impose for the time averaged values $\left|\partial \bar{C}_{i j}\right|, \bar{\nu}_{i j}$, and $\bar{\kappa}_{i j}$. Evaluated over $\partial C^{i j}(t)$, they should be carefully computed. In (Nkonga et al., 1994), both the cell's normals and the grid's velocity mean values determine the geometrical parameters which enforce the GCL. In (Farhat, 1995b), this is attained by means of a proper evaluation of the ALE fluxes using suited mesh configurations and grids velocities, showing also an equivalence with the former paper ideas. On the other hand, in (Dubuc et al., 2000), a scheme satisfying the GCL is proposed by tuning how the cell volume is evaluated. We also refer to (Farhat $e$ al., 2001) for examples of averagings satisfying the DGCL for the above time advancing scheme.

However, from an energy conservation point of view, this is not enough. While certainly it is for interior cells, additional care should be taken for boundary ones in order to keep the work performed on the fluid equal to the energy gain/loss of the fluid. While the GCL condition provides a way to calculate the mean normal vector and the mean normal mesh velocity $\boldsymbol{\beta}$ which does not introduce spurious effects due to the mesh movement, it says nothing about the flow velocity $\boldsymbol{u}$. In the interior nodes, both can be completely independent. But at the moving boundaries, that move at speed $\widehat{\boldsymbol{\beta}}$, some questions arise about the relationship between them. This analysis is completed in the next section.

\subsection{An integration on a facet satisfying $D G C L$}

As stated above in [21], the discretized space volume can be divided in control cells $C^{i}$, each of them limited by a faceted frontier $\partial C^{i}$. For a 3D problem, the boundary of an interior control cell is a polyhedron whose constitutive "facets" are triangles having as vertices:

- the middle of an edge $i j$ where $j$ is a neighbor of $i$,

- the centroid $D$ of $T$, one of the tetrahedra having $i$ and $j$ as vertices,

- the centroid $B$ of a face $i j k$ of T.

Two examples $\Sigma_{j k+}^{i}$ and $\Sigma_{k j+}^{i}$ are depicted in left part of Figure 4. The facet notation is the following: $\Sigma_{j k+}^{i}$ means "the facet corresponding to node $i$, relating it with node $j$ and with one of its sides lying on node $k$ 's median". By inspection it can be seen that there are only two possibilities for indices $(i, j, k)$ in this strict order. Therefore, labels + or - distinguish between both facets. 

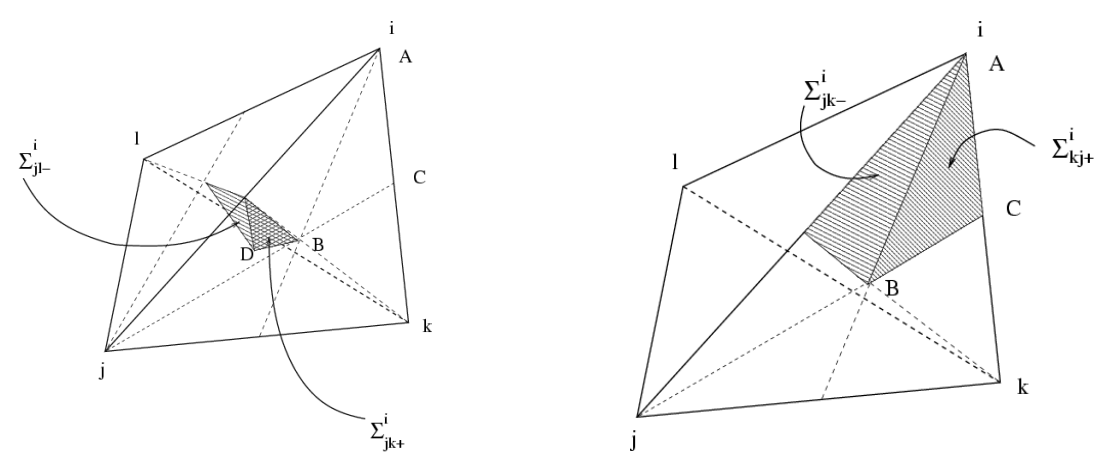

Figure 4. Tetrahedra and facets. Left, interior facets $\Sigma_{j l-}^{i}$ and $\Sigma_{j k+}^{i}$. Right, boundary facets $\Sigma_{j k-}^{i}$ and $\Sigma_{k j+}^{i}$

Therefore, Equation [22] can be pushed further:

$$
\partial C^{i}=\bigcup_{j \in V(i)} \partial C^{i j}=\bigcup_{j \in V(i)}\left(\bigcup_{k \in V(i)}\left(\Sigma_{j k+}^{i}+\Sigma_{k j-}^{i}\right)\right) .
$$

The DGCL can then be set for an arbitrary triangular facet. As said above, by enforcing this law, the time averaged values of the cell faceted surface $\left|\partial \bar{C}_{i j}\right|$, its normals $\overline{\boldsymbol{\nu}}_{i j}$, and its speeds $\bar{\kappa}_{i j}$ can be properly evaluated. In (Nkonga et al., 1994), this analysis is carried out for an interior node. Here we conduct the analysis for a boundary node still denoted by $i$. The boundary cell $C_{i}$ around $i$ is also limited by a polyhedron $\partial C_{i}$ made of triangular facets. The novelty is that, beside the interior facets already described, we have to consider the boundary facets.

A boundary facet is a triangle having as vertices:

- the mesh vertex $i$,

- the middle of $i j$ where $j$ is a neighboring boundary vertex,

- the centroid of a boundary face $i j k, k$ being a boundary vertex, which is a neighbor of $i$ and of $j$.

Two examples of such boundary facets are depicted in the right part of Figure 4. Let us consider any of these interior facets. It is a moving triangle changing its position between time $T^{n}$ and $T^{n+1}$, see Figure 5. It is shown in (Nkonga et al., 1994) that, for a given triangle $\Sigma_{\mathrm{NP}}^{\mathrm{M}}$, the mean normal $\bar{\nu}^{\Sigma}$ and the mean normal velocity $\bar{\kappa}^{\Sigma}$, defined above in [24] and [25] are

$$
\begin{aligned}
\overline{\boldsymbol{\nu}}^{\Sigma} & =\frac{1}{3}\left(\boldsymbol{\mu}^{\boldsymbol{n}}+\boldsymbol{\mu}^{\boldsymbol{n + 1}}+\boldsymbol{\mu}^{*}\right) \\
\bar{\kappa}^{\Sigma} & =\frac{\overline{\boldsymbol{\nu}}^{\Sigma}}{\left\|\overline{\boldsymbol{\nu}}^{\Sigma}\right\|} \cdot \boldsymbol{\beta}^{\Sigma},
\end{aligned}
$$




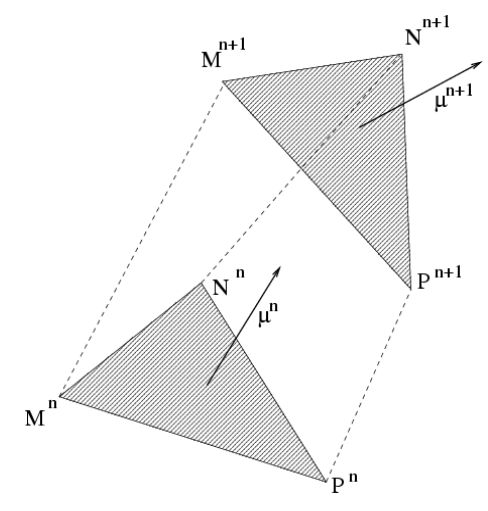

Figure 5. Triangle (representing a facet) $\Sigma_{\mathrm{NP}}^{\mathrm{M}}$ with its normal $\mu$

where $\boldsymbol{\mu}$ is the non-normalized exterior normal to the facet $\Sigma$, and the auxiliar variable $\boldsymbol{\mu}^{*}$ and the facet velocity $\boldsymbol{\beta}^{\Sigma}$ are defined as

$$
\begin{aligned}
\boldsymbol{\mu}^{*} & =\frac{\left(\boldsymbol{x}_{\mathrm{P}}^{n}-\boldsymbol{x}_{\mathrm{M}}^{n}\right) \wedge\left(\boldsymbol{x}_{\mathrm{N}}^{n+1}-\boldsymbol{x}_{\mathrm{M}}^{n+1}\right)}{4}+\frac{\left(\boldsymbol{x}_{\mathrm{P}}^{n+1}-\boldsymbol{x}_{\mathrm{M}}^{n+1}\right) \wedge\left(\boldsymbol{x}_{\mathrm{N}}^{n}-\boldsymbol{x}_{\mathrm{M}}^{n}\right)}{4} \\
\boldsymbol{\beta}^{\Sigma} & =\frac{\boldsymbol{\beta}_{\mathrm{M}}^{n}+\boldsymbol{\beta}_{\mathrm{N}}^{n}+\boldsymbol{\beta}_{\mathrm{P}}^{n}}{3} .
\end{aligned}
$$

For a given facet, the vertex velocities $\beta_{\mathrm{M}, \mathrm{N}, \mathrm{P}}$ are linearly interpolated from those at the corresponding tetrahedra nodes, because the related vertex $M, N$, or $P$ can be a node of the mesh, a mid-point segment, a face center or a tetrahedron center, noted respectively as A, C, B and D in Figure 4. For instance, for an interior facet like $\Sigma_{j l-}^{i}$ in Figure 4 (left), the facet velocity is (see (Nkonga et al., 1994))

$$
\boldsymbol{\beta}^{\Sigma, \mathrm{INT}}=\frac{1}{2}\left(\frac{13}{36}\left(\boldsymbol{\beta}_{i}+\boldsymbol{\beta}_{j}\right)+\frac{5}{36}\left(\boldsymbol{\beta}_{k}+\boldsymbol{\beta}_{l}\right)\right) .
$$

On the other hand, for a boundary facet like $\Sigma_{j k-}^{i}$, Figure 4 (right), we have:

$$
\boldsymbol{\beta}^{\Sigma, \mathrm{BOU}}=\frac{1}{2}\left(\frac{22}{36} \boldsymbol{\beta}_{i}+\frac{7}{36}\left(\boldsymbol{\beta}_{j}+\boldsymbol{\beta}_{k}\right)\right) .
$$

Since the integration is exact, these formulas allow for the DGCL. 


\subsection{The energy budget for the discretized problem}

Once the discretization method is set, the energy budget problem can be revisited, starting by considering the application of [21] to the flow energy equation. Let $\mathcal{E}_{i}=$ $\int_{C_{i}(t)} E$. Taking $v:=\rho e^{o}+\rho \frac{u^{2}}{2}=\rho e=E$ in [21], we obtain for an Euler problem

$$
\frac{d \mathcal{E}_{i}}{d t}+\int_{\partial C_{i}(t)} \boldsymbol{n}_{i} \cdot((E+p) \boldsymbol{u}-E \boldsymbol{\beta})=0
$$

Let us consider the control cells $C_{i}$ which share part of their facet boundaries with the moving wall boundary (a two-dimensional representation is given by Figure 6). For such cells we have

$$
\partial C_{i}(t) \cap \partial \Omega_{h}(t)=\partial \widehat{C}_{i}(t) \neq \emptyset
$$

It can be expected that in order to maintain the impermeability condition, the normal boundary velocity at the intersection with the moving wall boundary should be equal to the flow velocity.

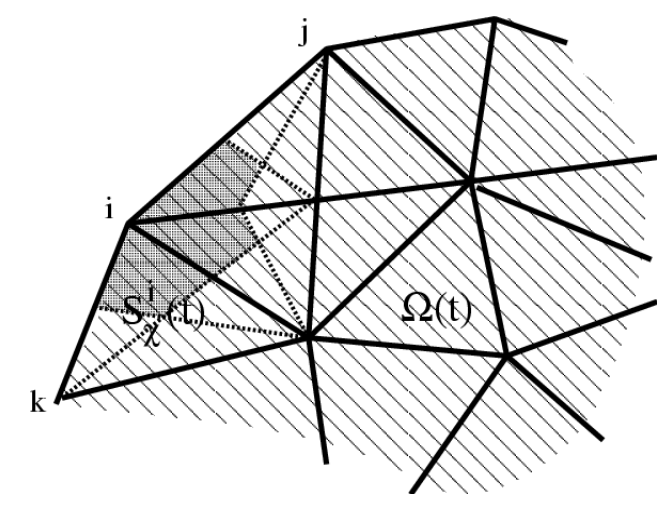

Figure 6. Two-dimensional representation of a cell on the moving wall boundary

The previous boundary cells $C_{i}$ have both an "interior" part and a "moving wall" part, $\partial C_{i}=\partial C_{\mathrm{O}}^{i}+\partial C_{\mathrm{MW}}^{i}$, where

$$
\begin{gathered}
\partial C_{\mathrm{MW}}^{i}=\partial C_{i}(t) \cap\left(\bigcup_{\Sigma_{\mathrm{MW}}, i \in \Sigma_{\mathrm{MW}}} \Sigma_{\mathrm{MW}}\right) \\
\partial C_{\mathrm{O}}^{i}=\partial C_{i}(t) \backslash \partial C_{\mathrm{MW}}^{i} .
\end{gathered}
$$

where $\Sigma_{\mathrm{MW}}$ denote the set of the facets which belong to the moving part of $\partial \Omega_{h}(t)$. It should be remarked that in this case, [22] which expresses that the cell boundary is 
the union of interfaces with neighboring cells is not true because it is associated now to a boundary node. Instead, [34] can be written as

$$
\begin{aligned}
\frac{d \mathcal{E}_{i}}{d t} & +\int_{\partial C_{\mathrm{O}}^{i}} \boldsymbol{n}_{i} \cdot(E \boldsymbol{w}+p \boldsymbol{u}) \\
& +\int_{\partial C_{\mathrm{MW}}^{i}} \boldsymbol{n}_{i} \cdot(\widehat{E} \widehat{\boldsymbol{w}}+\widehat{p} \widehat{\boldsymbol{u}})=0
\end{aligned}
$$

where

$$
\boldsymbol{w}=\boldsymbol{u}-\boldsymbol{\beta} .
$$

This is the velocity difference between the mesh and the flow field at node $i$. As said before, in a general ALE formulation, mesh speed and flow velocities are not related, except at the moving boundaries. By introducing a numerical flux function as [26], Equation [36] becomes

$$
\begin{aligned}
\frac{d \mathcal{E}_{i}}{d t} & +\sum_{j \in V(i)}\left|\partial C_{i j}(t)\right| \Phi_{\mathrm{O}}\left(W_{i}, W_{j}, \boldsymbol{\nu}_{i j}(t), \kappa_{i j}(t)\right) \\
& +\sum_{\Sigma, i \in \Sigma}\left|\partial \widehat{C}_{i}(t) \cap \Sigma\right| \Phi_{\mathrm{MW}}\left(\widehat{W}^{\Sigma}, \widehat{\boldsymbol{w}}^{\Sigma}, \widehat{\boldsymbol{\nu}}^{\Sigma}(t), \widehat{\kappa}^{\Sigma}(t)\right)
\end{aligned}
$$

where $W$ is the flow solution vector and $\Sigma$ is a triangular facet which belongs to the moving wall boundary.

Equation [38] is the core of the problem. For the interior cells, the second summation vanishes and the first summation over the "internal" segments completes the cell boundaries. But for the boundary cells, the second summation introduces a substancial difference. How is evaluated $\widehat{\boldsymbol{w}}$ on a moving boundary facet? Once integrated in time and summed up to consider the extensive energy $\mathcal{E}$ of the whole domain, Equations [36] and [38] will give the amount of energy gained or loss by the discretized system [11], that could be in turn compared with the work performed on it [16]. Let us take [36]. Equation [11] states that

$$
\begin{aligned}
\left.\Delta \mathcal{E}\right|_{t_{1}} ^{t_{2}} & =\int_{t_{1}}^{t_{2}} \frac{d \mathcal{E}}{d t}=\int_{t_{1}}^{t_{2}} \sum_{i}^{n_{c}} \frac{d \mathcal{E}_{i}}{d t}=\left.\sum_{i=1}^{n_{c}} \mathcal{E}_{i}\right|_{t_{1}} ^{t_{2}} \\
\left.\mathcal{E}_{i}\right|_{t_{1}} ^{t_{2}} & =-\int_{t_{1}}^{t_{2}} \int_{\partial C_{\mathrm{MW}}^{i}} \boldsymbol{n}_{i} \cdot(\widehat{E} \widehat{\boldsymbol{w}}+\widehat{p} \widehat{\boldsymbol{u}})
\end{aligned}
$$

where the summation runs over all cells $i$ of the mesh. The terms corresponding to the energy interchange between interior cells cancel each other and the remaining terms are those corresponding to the boundary contribution. 
On the other hand, the work performed on the fluid by the moving boundary is

$$
\begin{aligned}
\left.W\right|_{t_{1}} ^{t_{2}} & =-\int_{t_{1}}^{t_{2}} \int_{\partial \Omega(t)} \widehat{p} \widehat{\boldsymbol{\beta}} \cdot \boldsymbol{n} \\
& =-\int_{t_{1}}^{t_{2}} \sum_{i}^{n_{c}} \int_{\partial C_{\mathrm{MW}}^{i}} \widehat{p} \widehat{\boldsymbol{\beta}} \cdot \boldsymbol{n}_{i} .
\end{aligned}
$$

From Equations [39] and [40], as in the DGCL case, several questions on how are approximated the boundary values and fluxes must be considered.

\section{Global conservation}

\subsection{Theoretical statement}

It has been shown that assuming that the DGCL is satisfied inside the mesh, the issue of the total energy conservation is restricted to the discrete equations on boundary cells, and particularly the contribution of boundary fluxes. In order to fix the ideas, recall that:

- The solid moving boundary $\partial \Omega(t)$ moves at speed $s$ defined at any vertex.

- The flow moves at velocity $\boldsymbol{u}$ wherever, and particularly at velocity $\widehat{\boldsymbol{u}}$ defined at any vertex of $\partial \Omega(t)$.

- The mesh moves at speed $\boldsymbol{\beta}$ wherever, and particularly at speed $\widehat{\boldsymbol{\beta}}$ defined at any vertex of $\partial \Omega(t)$.

- The difference between mesh speed and velocity flow is $\boldsymbol{w}$, and particularly $\widehat{\boldsymbol{w}}$ defined at any vertex of $\partial \Omega(t)$.

Having this in mind, together with both geometric and energy conservation as set by GCL and Equations [39] - [40], it is observed that for any boundary cell, there exists a great deal of approximations for computing the boundary flows. Nevertheless, not all of them are properly conservative ones. On one hand, the DGCL has to be accomplished. Equations [32] and [33] tell us how to compute the facet velocity once we know the mesh velocities of each interior or boundary node $i$, noted $\boldsymbol{\beta}_{\boldsymbol{i}}$ and $\widehat{\boldsymbol{\beta}_{\boldsymbol{i}}}$ respectively. In the interior nodes, $\boldsymbol{\beta}_{\boldsymbol{i}}$ can be arbitrarily assigned. We recall that the in/output boundaries are not moving. However, for those lying at the moving boundaries, $\widehat{\boldsymbol{\beta}_{\boldsymbol{i}}}$ cannot be arbitrarily set. The [31] needs be used to compute the mean values. Independently of the use of [33] in [31], the second conservation principle involved here must be satisfied. We summarize this as follows. Let us consider the "moving wall" boundary $\partial C_{\mathrm{MW}}^{i}$ made of boundary facets (see Figure 4, right). Then, we can write the following lemma: 
Lemma: A GCL-compatible integration of the energy flux through the "moving wall" boundary $\partial C_{\mathrm{MW}}^{i}$ i.e. an integration satisfying [29], is obtained when Equation [39] is approximated as follows:

$$
\left.\mathcal{E}_{i}\right|_{t_{1}} ^{t_{2}}=-\int_{t_{1}}^{t_{2}} \int_{\partial C_{\mathrm{MW}}^{i}}\left(\widehat{E}_{i} \widehat{\boldsymbol{w}}_{i}+\widehat{p}_{i} \widehat{\boldsymbol{u}}_{i}\right) \cdot \boldsymbol{n}_{i}
$$

where, for each boundary facet $\Sigma$ which intersects with $\partial C_{\mathrm{MW}}^{i}$, the GCL based time-space integration of the mesh normal and normal velocities is computed using [31] and [33], and where facet mean values $\widehat{p}_{i}$ of the pressure and $\widehat{E}_{i}$ of the total energy are used. $\square$

Definition: A space-time integration scheme will be total energy conservative if the energy loss (or gain) given by the discretized counterpart of Equation [39] is exactly the work produced (or received), defined by the discretized counterpart of Equation [40], that is if:

$$
\left.\Delta \mathcal{E}\right|_{t_{1}} ^{t_{2}}=\left.W\right|_{t_{1}} ^{t_{2}}=-\int_{t_{1}}^{t_{2}} \sum_{i=1}^{n_{c}} \int_{\partial C_{\mathrm{MW}}^{i}} \widehat{p}_{i} \widehat{\boldsymbol{\beta}}_{i} \cdot \boldsymbol{n}_{i}
$$

where all the above quantities are time and space discretized ones.

In particular, for a GCL-compatible scheme, the previous integral over each facet of $\partial C_{\mathrm{MW}}^{i}$ is computed with a time-space integration based on [31] and [33], and a facet mean value $\widehat{p}_{i}$ of the pressure. From the above lemma and definition, we can build a simple conservative scheme in the sense that it satisfies both the GCL and the total energy conservation.

Proposition: Let consider a scheme defined by [28],[29],[31],[32],[33] which satisfies the conditions of the above lemma, that is [41], where, for each moving facet $\Sigma_{\mathrm{MW}}$, the mean on that facet of the flow normal velocity $\widehat{\boldsymbol{u}}_{i} \cdot \boldsymbol{n}_{i}$ is replaced by the mean on the facet of the mesh normal velocity $\widehat{\boldsymbol{\beta}}_{i} \cdot \boldsymbol{n}_{i}$. Then the scheme satisfies [42], i.e. is total energy conservative. 
Proof: The energy conservation stated by Lemma 2 is satisfied since:

$$
\begin{aligned}
\left.\Delta \mathcal{E}\right|_{t_{1}} ^{t_{2}} & =-\sum_{i=1}^{n_{c}} \int_{t_{1}}^{t_{2}} \int_{\partial C_{\mathrm{MW}}^{i}}\left(\widehat{E}_{i} \widehat{\boldsymbol{w}}_{i}+\widehat{p}_{i} \widehat{\boldsymbol{u}}_{i}\right) \cdot \boldsymbol{n}_{i} \\
& =-\sum_{i=1}^{n_{c}} \int_{t_{1}}^{t_{2}} \int_{\partial C_{\mathrm{MW}}^{i}} \widehat{p}_{i} \widehat{\boldsymbol{u}}_{i} \cdot \boldsymbol{n}_{i} \\
& =-\sum_{i=1}^{n_{c}} \int_{t_{1}}^{t_{2}} \int_{\partial C_{\mathrm{MW}}^{i}} \widehat{p}_{i} \widehat{\boldsymbol{\beta}}_{i} \cdot \boldsymbol{n}_{i} \\
& =\left.W\right|_{t_{1}} ^{t_{2}}
\end{aligned}
$$

The previous condition on the flow and mesh normal velocities can be achieved by requiring that $\widehat{\boldsymbol{w}}_{i} \cdot \boldsymbol{n}_{i}=\left(\widehat{\boldsymbol{u}}_{i}-\widehat{\boldsymbol{\beta}}_{i}\right) \cdot \boldsymbol{n}_{i}=0$ for each boundary node. $\square$

\subsection{Impact on the numerical algorithm}

Starting from an ALE conservative discretization of the Euler equations, a total energy conservative method is obtained by:

- ensuring that the DGCL is satisfied on internal cells interfaces and on boundary cell surfaces. In our case, this is defined by [28],[29],[31],[32],[33],

- using the particular boundary quadrature defined by [41] using $\widehat{\boldsymbol{\beta}}_{i} \cdot \boldsymbol{n}_{i}$ for $\widehat{\boldsymbol{u}}_{i} \cdot \boldsymbol{n}_{i}$.

We have assumed that time integration is explicit. Total energy conservation also applies for a non-linear implicit advancing.

Remark: In the case where the fluid model is coupled with a structural one, a notable consequence of the total energy conservation principle involved here is the following: any transmission of the fluid pressure consistent with the above integration (use of the hat integration values) will allow a perfect energy budget, that is, any Joule lost by the fluid will be gained by the structure and vice versa. In practice, this situation is not obtained with time advancing schemes weakly coupling the two materials. In that latter case, energy conservation can be satisfied up to a higher order error (Piperno et $a l ., 2000)$. Exact energy conservation is attainable with the so-called strongly coupled monolithic time advancing schemes.

\section{Numerical experiments}

The main output of the present work is a discrete model satisfying a strict total energy conservation. In the volumic part of the discretisation this conservation is ensured by the Discrete Geometric Conservation Law, a property recommended in many papers and satisfied by many softwares, in particular by the fluid-structure methodology developped by Farhat and co-workers (Farhat, 1995b). In (Farhat, 1995b), the 
aeroelastic coupled system is computed by a partitioned procedure for the solution of fluid-structure interaction problems (Farhat et al., 2000). This partitioned procedure is implicit and linearly unconditionally stable. For the boundary part of the discretisation, the present paper proposes a new quadrature. We have introduced this new quadrature in that software as a new option. The flow field can be solved using either the "fluid energy conservative" quadrature (Scheme 1) described in the previous section, or its "fluid energy non-conservative" counterpart (Scheme 2) in which the boundary flux is computed with a second-order accurate quadrature. Note that both options satisfy the DGCL property.

Impact on conservation: Let us consider a box $[0, L] \times[0,1] \times[0,1]$ containing a mass of gas, with slip condition on faces. Its length $L$ is smoothly varied in time with a sine oscillation. Then the integral $E(t)$ of total energy should vary as the sum $S(t)$ of its initial value and of the work given to this system, i.e. the space-time integral of pressure on the moving face, $x=L(t)$. We have choosen a coarse cartesian mesh of $21 \times 21 \times 21$ vertices. The finite volume approximation is upwind biased (third-order accurate for linear systems and uniform meshes). The time advancing is a four-stage explicit Runge-Kutta. With this time advancing, the novel scheme (Scheme 1) should conserve exactly the energy. Scheme 2 should not. After computation, we check that Scheme 1 is perfectly conservative (up to floating point round off errors). For Scheme 2, we present in Figure 5 the two outputs which should be equal, together with their difference. The maximum relative difference is about $2.5 \%$. Scheme 2 is rather close to be conservative, we think this is because it satisfies the volumic DGCL, but produces still a non-negligible error.
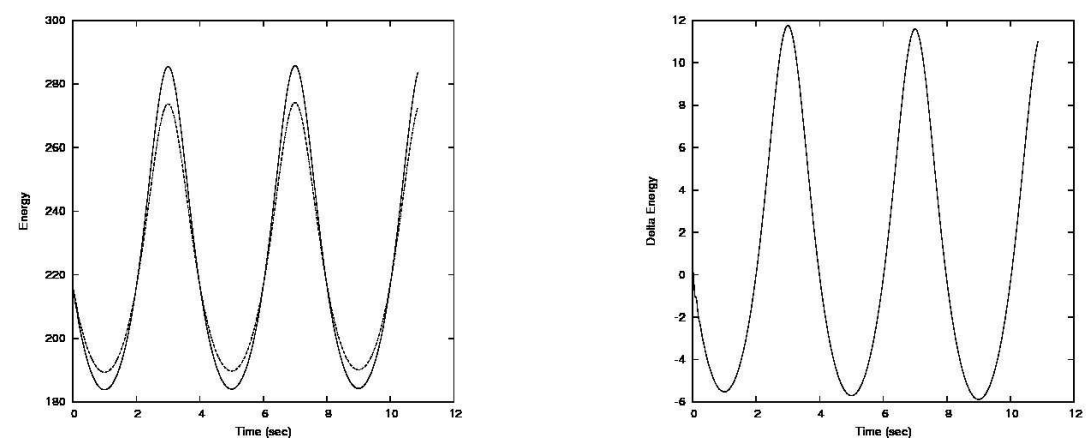

Figure 7. Cubic piston calculation for Scheme 2: left, comparison of total energy evolution with the evolution of adding the initial total energy and the work yielded to gas. Right, the difference of these two outputs as a function of time

Impact on stability: We propose now a computation showing that the exact fluid energy conservation property has also a small but certain influence on the actual stability limit of a coupled fluid-structure simulation. This can be intuitively explained for an isolated system by the fact that when total physical energy is conserved, it remains bounded. We choose a flow studied many times in the litterature. In several publica- 
tions of the authors, this flow is used for measuring the progress (stability, accuracy, efficiency) of the numerical methods, see (Farhat, 1995a; Farhat et al., 2000; Piperno et al., 2000; Cournède et al., 2006). We consider the flutter analysis of the AGARD Wing 445.6 which has been conducted experimentally for various flow conditions by Yates (Yates, 1987). The test-case investigated in this section concerns the 2.5 weakened model 3, and the freestream conditions are set to $M_{\infty}=0.901, \rho_{\infty}=1.117 \times 10^{-7}$ slugs $/ \mathrm{in}^{3}$ and $p_{\infty}=10.0 \mathrm{slugs} /\left(\mathrm{s}^{2} \times\right.$ in $)$. Yates indicates that the conditions of this case are inside the flutter stability domain for the considered wing (i.e. no flutter). The three-dimensional unstructured tetrahedral CFD mesh contains 22014 vertices. The structure of the wing is discretized by a thin plate finite element model which contains 800 triangular composite shell elements (Farhat et al., 2000). It yields natural mode shapes and frequencies that are similar to those derived experimentally (Yates, 1987). Both fluid and structure are advanced in time with an implicit scheme. A weak fluidstructure coupling is used as in (Farhat et al., 2000). In contrast to a monolithic strong coupling scheme, the weak coupling does not conserve exactly the kinetic energy between fluid and structure, but only at a higher order of accuracy. The purpose of the experiment is to examine the impact of the proposed spatial total energy conservation method in these conditions.
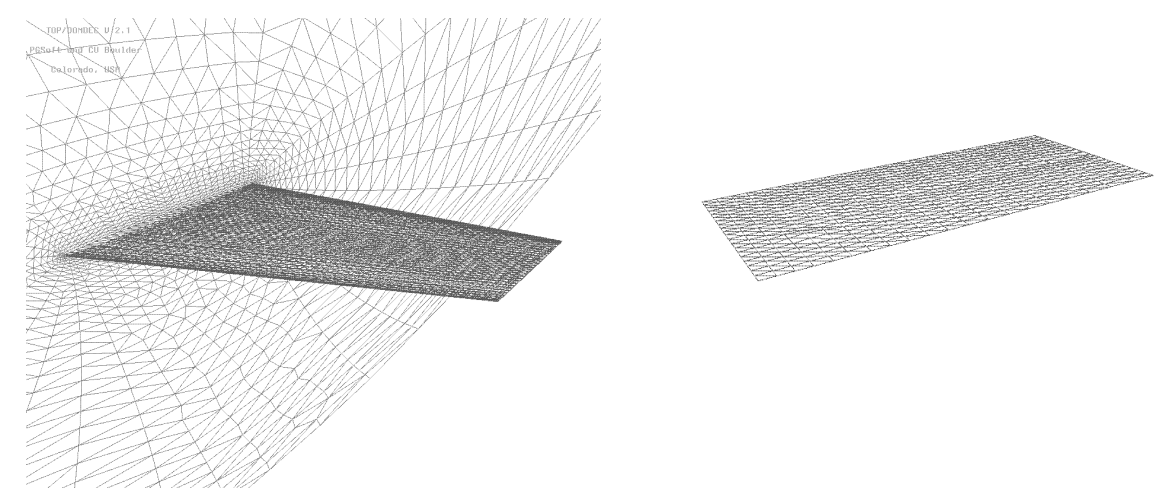

Figure 8. AGARD-wing test case: view of fluid mesh and structure mesh

The finite element structural model is perturbated along its first bending mode, and a steady state solution is computed around the deformed configuration of the wing. Next this perturbation is used as initial condition, and the aeroelastic response of the wing is computed by advancing in time the coupled system. In this flutter analysis, the dimensional fluid-structure coupling time-step is set either to $\Delta t=7.5 \times 10^{-4} \mathrm{~s}$ or $\Delta t=5 \times 10^{-4} \mathrm{~s}$, which correspond respectively to CFL numbers of about 600 and 900. These time-steps correspond to sampling the period of the damped oscillations which characterize the lift representative of the wing aeroelastic response in 90 and 140 time intervals, respectively.

In Figures 10 and 11, we depict the lift histories for the "fluid energy conservative" scheme and its energy non-conservative counterpart respectively. These figures 


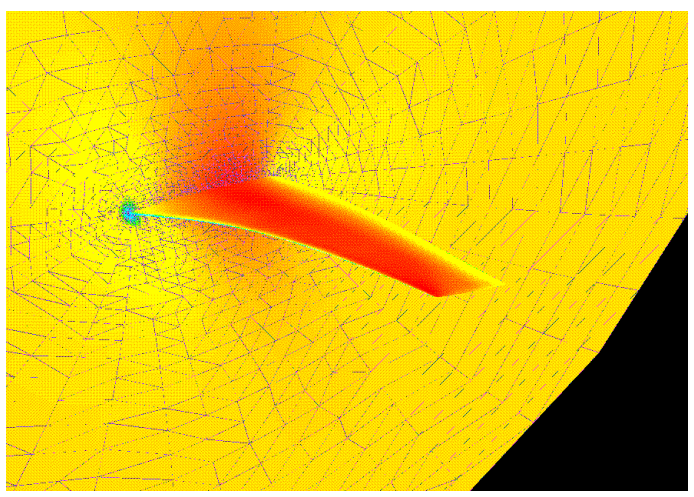

Figure 9. AGARD-wing test case: instantaneous Mach number field

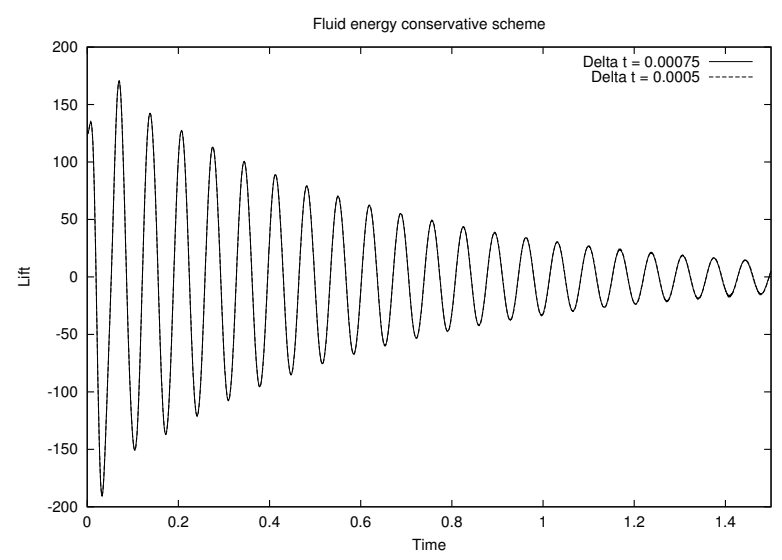

Figure 10. Lift histories predicted by the "fluid energy conservative" scheme for the flutter analysis of the AGARD Wing 445.6

indicate that for a time step $\Delta t=5 \times 10^{-4} \mathrm{~s}$, both schemes predict correctly the flow and in particular that the wing does not flutter for the given freestream conditions. For a time step of $\Delta t=7.5 \times 10^{-4} \mathrm{~s}$, the answer produced by the "fluid energy conservative" scheme is very close to the previous ones. This shows in particular, that the time convergence is good and the higher time step is just a $50 \%$ more efficient option. On the contrary, the "fluid energy non-conservative" scheme predicts, after some time, an instable behavior. Since for smaller time steps the answer is good, the instability is 


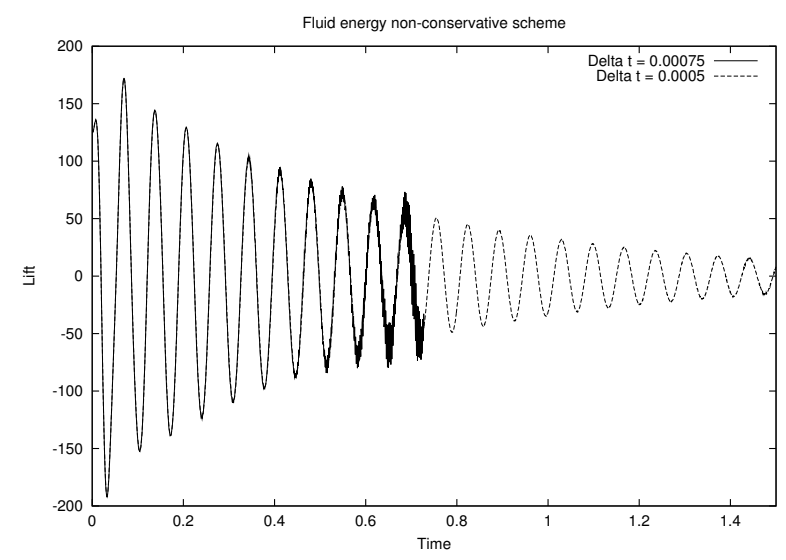

Figure 11. Lift histories predicted by the "fluid energy non-conservative" scheme for the flutter analysis of the AGARD Wing 445.6

probably of numerical origin. The "fluid energy conservative" scheme is also near its practical stability limit (about $\Delta t=8 . \times 10^{-4} \mathrm{~s}$ ) but enjoys a supplement of stability thanks to a small modification of the boundary energy flux.

\section{Conclusion}

Three important classes of conservation relations need be satisfied by numerical models when applied to nonlinear/unsteady interactions between a compressible fluid and a structure: the usual conservations (mass, moment, energy) in each medium, the Geometric Conservation Law, in the fluid, and the conservation of total energy.

We have shown that for the fluid part, conservation of total energy can be satisfied by some particular spatial discretisations. To get this conservation, the Discrete Geometric Conservation Law is a paramount ingredient, but it needs to be extended to the domain boundary. Further, a particular class of quadrature for the velocity needs to be applied.

The new scheme permits an exact transmission of the fluid energy, from the total fluid energy variable to the total structure energy, as in the physical differential model.

We have highlighted the importance of the fluid total energy conservation property on a simple piston problem. Even for a very smooth flow, on a short time interval, energy error is of several percents with the previous scheme while it is zero with the new formulation. 
We have also highlighted the impact of the fluid total energy conservation property on the stability of a weakly coupling implicit time advancing. This is illustrated by computing a flutter analysis of the AGARD Wing 445.6. Increase in stability is about $50 \%$.

Schemes enjoying these conservation properties should produce more reliable results for violent transient problems where energy transfers of high local (in space or time) strength occur. They should be very useful to evaluate more accurately energy budgets when several small energy losses are in competition (radiation,...). In future works, we plan to continue various numerical experiments for measuring these improvements and introduce the proposed numerical technology into more complex models, in particular involving dissipative effects.

\section{Acknowledgements}

The authors thanks Dr Stephen Wornom for helpfull discussions and for his contribution to the first numerical experiment. The authors wish to thank the following computing centers: IDRIS at Saclay, CINES at Montpellier and GRID5000.

\section{References}

Cournède P.-H., Koobus B., Dervieux A., "Positivity statements for a Mixed-Element-Volume scheme on fixed and moving grids" , Revue Européenne des Eléments Finis, vol. 15, n 7-8, p. 767-799, 2006

Dubuc L., Cantariti F., Woodgate M., Gribben B., Badcock K., Richards B., "A grid deformation technique for unsteady flow computations", journal = "Int. J. Num. Meth. Fluids." , , vol. 32, p. 285-311, 2000.

Fanion T., Fernandez M., Le Tallec P., "Deriving Adequate Formulations for Fluid Structure Interaction Problems : from ALE to Transpiration", Revue Européenne des Eléments Finis, vol. 9, n 6-7, p. 681-708, 2000.

Farhat C., "High performance simulation of coupled nonlinear transient aeroelastic problems", Special course on parallel computing in CFD. $n^{\circ}$ R-807, NATO AGARD Report, October, 1995a.

Farhat C., "High performance simulation of coupled nonlinear transient aeroelastic problems", type = "Special course on parallel computing in CFD", Technical Report n ${ }^{\circ}$ R-807, NATO AGARD Report, October, 1995b.

Farhat C., Geuzaine P., Grandmont C., "The Discrete Geometric Conservation Law and the nonlinear stability of ALE schemes for the solution of flow problems on moving grids" , $J$. of Computational Physics, vol. 174, p. 669-694, 2001.

Farhat C., Lesoinne M., "Two efficient staggered procedures for the serial and parallel solution of three-dimensional nonlinear transient aeeroelastic problems" , Comp. Meths. Appl. Mech. Eng., vol. 182, n 3-4, p. 499-515, 2000.

Farhat C., Lesoinne M., Le Tallec P., "Load and motion transfer algorithms for Fluid/Structure iteraction problems with Non-Matching discrete interfaces: momentum and energy conservation, optimal discretization and application to aeroelasticity", journal = "Comp. Meth. Appl. Mech. Eng." , , vol. 157, p. 95-114, 1998. 
Fernandez M., Le Tallec P., "Un nouveau problème spectral en interaction fluide-structure avec transpiration = A new spectral problem in fluid-structure interaction with transpiration" , Comptes rendus de l'Académie des sciences. Série 1, Mathématique, vol. 334, n 2, p. 167$172,2002$.

Grandmont C., Maday Y., "Fluid-structure interaction: a theoretical point of view" , Revue Européenne des Eléments Finis, vol. 9, n 6-7, p. 633-654, 2000.

Guillard G., Farhat C., "On the significance of the geometric conservation law for flow computation on moving meshes", Comput. Meth. Appl. Mech. Eng., vol. 190, p. 1467, 2000.

Hirt C. W., Amsden A. A., Cook J. L., "An arbitrary Lagrangian-Eulerian computiong method for all flow speeds" , Journal of Computational Physics, vol. 14, p. 227-253, 1974.

Koobus B., Farhat C., "Second-order time accurate and geometrically conservative implicit schemes for flow computation on unstructured dynamic meshes", Comput. Meth. Appl. Mech. Eng., vol. 170, p. 103, 1999.

Landau L., Lifshitz E., Fluid Mechanics, 2nd. edn, Butterworth - Heinemann, 1987.

Le Tallec P., Fernandez M., "Calcul d'écoulements en domaines déformables : de l'ALE à la transpiration (in french)" , La Houille Blanche, vol. 6, p. 51-77, 2000.

Le Tallec P., Gerbeau J.-F., Hauret P., Vidrascu M., "Fluid structure interaction problems in large deformation", Comptes Rendus, Mécanique, vol. 333, n 12, p. 910-922, 2005.

Lesoinne M., Farhat C., "Geometric conservation laws for flow problems with moving boundaries and deformable meshes and their impact on aeroelastic computations" , Comp. Meth. Appl. Mech. Eng., vol. 134, p. 71-90, 1996.

Leyland P., Carstens V., Blom F., Tefy T., "Fully coupled fluide-structure algorithms for aeroelasticity and forced vibration induced flutter", Revue Européenne des Eléments Finis, vol. 9, n 6-7, p. 763-803, 2000.

Mani S., "Truncation error and energy conservation for fluid structure interactions", Comput. Methods Appl. Mech. Engrg., vol. 192, n 43, p. 4769-4804, 2003.

Nkonga B., Guillard H., "Godunov type method on non-structured meshes for threedimensional moving boundary problems", Comput. Methods Appl. Mech. Eng., vol. 113, $\mathrm{n}^{\circ}$ 1-2, p. 183-204, 1994.

Piperno S., Farhat C., "Design of efficient partitioned procedures for the transient solution of aeroelastic problems", Revue Européenne des Eléments Finis, vol. 9, n 6-7, p. 655-680, 2000 .

Thomas P., Lombard C., "Geometric conservation law and its application to flow computations on moving grids" , AIAA Journal, vol. 17, p. 1030-1037, 1979.

Yates E., "AGARD standard aeroelastic configuration for dynamic response, candidate configuration I. - Wing 445.6" , NASA TM-100492, 1987.

Received: 10 February 2009

Accepted: 23 February 2010 
